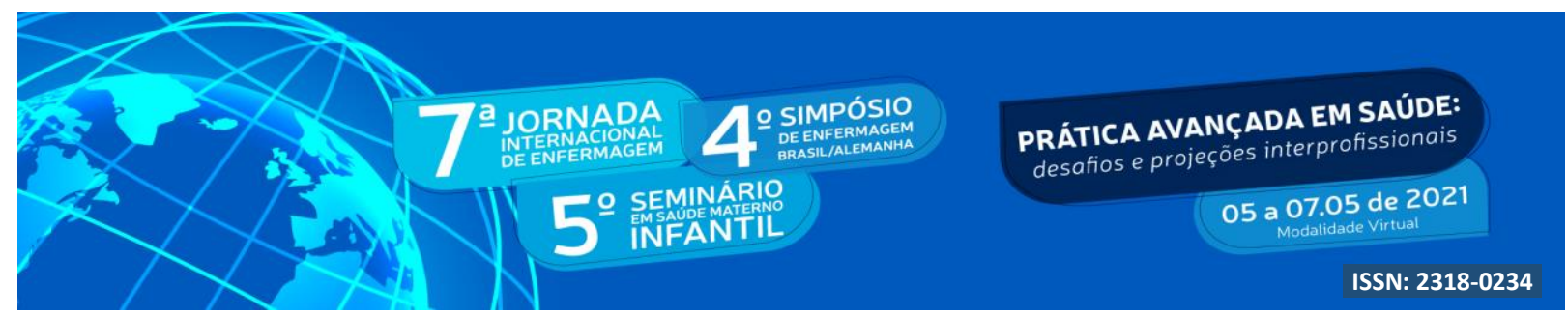

DOI: http://doi.org/10.48195/jie2021-100

\title{
INTERVENCC̃̃O EDUCATIVA COMO FERRAMENTA DE PREVENÇÃO À VIOLÊNCIA OBSTÉTRICA: UMA REVISÃO INTEGRATIVA ${ }^{1}$
}

\author{
Larissa Beatriz Francisca de Souza ${ }^{2}$; Maria de Lourdes Alves da Cruz ${ }^{3}$; Sidney Bruno \\ Lima da Silva"; Ana Luiza Santos Quirino5; Renata Marinho Fernandes ${ }^{6}$ Ana Luisa \\ Brandão de Carvalho Lira ${ }^{7}$
}

\begin{abstract}
RESUMO
Objetivou-se identificar o perfil das intervenções educativas voltadas para a prevenção da violência obstétrica presentes na literatura. Trata-se de uma revisão integrativa da literatura, realizada nas fontes de dados Cumulative Index to Nursing and Allied Health Literature, Scopus Content Overview, Literatura Latino-Americana e do Caribe em Ciências da Saúde e PubMed. Foram obtidos 817 artigos, selecionando-se 12 para compor a amostra. Em relação aos estudos incluídos, a maioria eram estudos nacionais $(33,3 \%)$ e do tipo experimental $(66,7 \%)$. Evidenciou-se que as intervenções educativas foram direcionadas para as gestantes, parturientes, graduandos, profissionais de saúde e/ou população geral. Estas repercutem no empoderamento das mulheres, favorecendo a autonomia sobre o parto e apropriação dos seus direitos. Também favoreceram a reflexão crítica da realidade, impactando na transformação da prática assistencial. Conclui-se que as intervenções educativas têm potencial para prevenir casos de violência obstétrica por meio da aquisição e fortalecimento da construção do conhecimento.
\end{abstract}

Palavras-chave: Educação; Educação em Saúde; Violência contra mulheres; Violência obstétrica.

\begin{abstract}
The objective was to identify the profile of educational interventions aimed at preventing obstetric violence in the literature. This is an integrative literature review, carried out on the data sources Cumulative Index to Nursing and Allied Health Literature, Scopus Content Overview, Latin American and Caribbean Literature on Health Sciences and PubMed. 817 articles were obtained, 12 were selected to compose the sample. Regarding the included studies, most were national $(33.3 \%)$ and experimental (66.7\%) studies. Educational interventions were directed at pregnant women, parturients, undergraduates, health professionals and / or the general population. These have an impact on the empowerment of women, favoring autonomy over childbirth and appropriation of their rights. They also favored a critical reflection of reality, impacting on the transformation of care practice. It is concluded that educational interventions have the potential to prevent cases of obstetric violence through the acquisition and strengthening of knowledge construction.
\end{abstract}

\footnotetext{
${ }^{1}$ Revisão integrativa da literatura

${ }^{2}$ Estudante do Curso de Enfermagem. UFRN. E-mail: larissa.beatriz@ufrn.edu.br

${ }^{3}$ Estudante do Curso de Enfermagem. UFRN. E-mail: maria_lourdes@ @otmail.com

${ }^{4}$ Estudante do Curso de Enfermagem. UFRN. E-mail: sidney.natal10@gmail.com

${ }^{5}$ Estudante do Curso de Enfermagem. UFRN. E-mail: luizasantos699@gmail.com

${ }^{6}$ Mestra em Enfermagem. UFRN. E-mail: remarifernandes@gmail.com

${ }^{7}$ Orientadora. Doutora em Enfermagem. UFRN. E-mail: analuisa_brandao@yahoo.com.br
} 


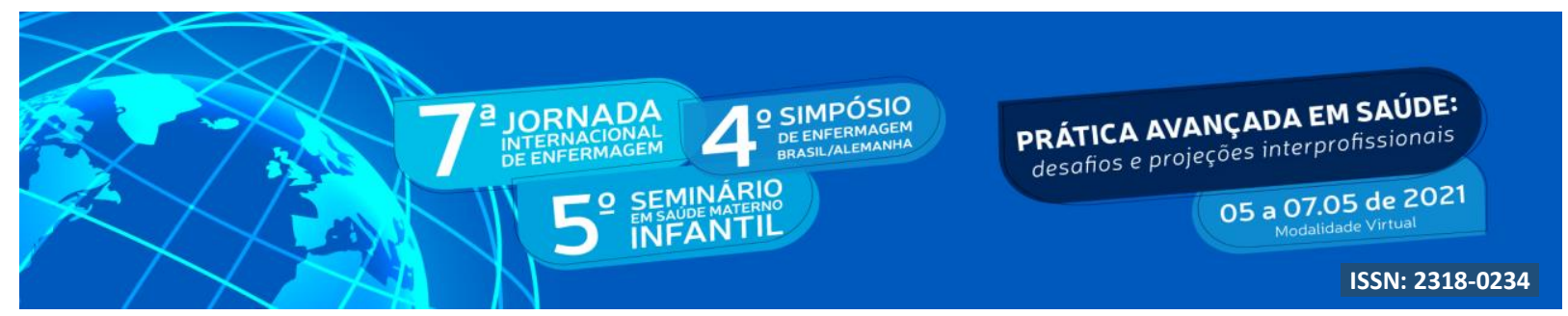

Key Words: Education; Health Education; Violence against women; Obstetric violence.

\section{INTRODUÇÃ̃O}

Até o final do século 18, o parto e o nascimento eram vistos como um fenômeno fisiológico e feminino, considerado um ritual de mulheres, no qual era acompanhado em domicílio pelas parteiras (STONE, 2016). No entanto, com a padronização da medicina ocidental, este fenômeno foi se tornando um ato médico, masculino e intervencionista, atribuindo novos significados à assistência obstétrica (STONE, 2016).

Diante desse cenário, o protagonismo que antes pertencia à mulher, atualmente é direcionado à figura médica, considerada a autoridade durante a assistência ao parto (BEGLEY et al., 2019). Como resultado, o cuidado às gestantes e puérperas têm sido substituído pela supervalorização da tecnologia, excesso de medicalização e imposição do saber técnico-científico, favorecendo a existência de diferentes formas de desrespeito às mulheres nos serviços de saúde (JARDIM; MODENA, 2018).

Para identificar qualquer ato de abuso e desrespeito causado pelos profissionais ou instituições às gestantes, puérperas ou a seu bebê, foi proposto o uso da terminologia violência obstétrica (VO), atualmente reconhecida como um problema de saúde pública pela Organização Mundial de Saúde (OMS) (OMS, 2014; SADLER et al., 2016). Tal termo engloba maus-tratos físicos e verbais, intervenções médicas desnecessárias e desrespeito à autonomia da mulher, contribuindo com maiores índices de morbimortalidade materna e neonatal (SADLER et al., 2016; MENEZES et al., 2020).

Em relação a sua ocorrência, vale ressaltar que a VO está presente em todo o mundo, sendo prevalentemente comum em países de baixa e média renda, como Tanzânia e Brasil, em que as taxas são maiores do que 70\% (SANDO et al., 2017). Outrossim, salienta-se os altos números de intervenções desnecessárias durante o parto, ocorrendo em $70 \%$ dos casos na Europa e acima de 40\% em países da América Latina (SADLER et al., 2016).

Logo, diante de um cenário de violação dos direitos humanos, faz-se necessário a criação de intervenções capazes de garantir assistência humanizada durante o parto e nascimento, no qual as demandas biopsicossociais das gestantes e parturientes sejam atendidas (JARDIM; MODENA, 2018). Nesse sentido, destaca-se a educação, como 


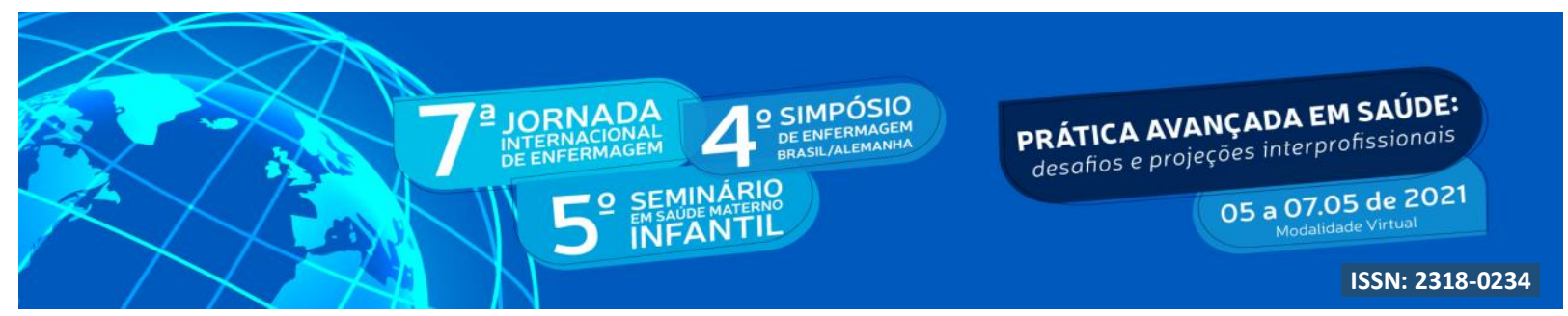

ferramenta fundamental para o fortalecimento de ações transformadoras contra a violência obstétrica e responsabilização do cuidado (MENEZES et al., 2020).

Portanto, o presente estudo se justifica, em razão da importância das intervenções educativas para prevenção e redução dos casos de violência obstétrica, de modo a contribuir para com a saúde e bem-estar das mulheres, principalmente, em um período da vida de maior vulnerabilidade e com diferentes mudanças fisiológicas e psicossociais.

\section{OBJETIVO}

Identificar o perfil das intervenções educativas voltadas para a prevenção da violência obstétrica presentes na literatura.

\section{METODOLOGIA}

Trata-se de uma revisão integrativa da literatura, fundamentada nas recomendações do Preferred Reporting Items for Systematic Reviews and Meta-Analyses (PRISMA). O protocolo de pesquisa foi revisado e registrado na plataforma International Prospective Register of Systematic Reviews (PROSPERO) (CRD42021238323).

Para direcionar o estudo, foi estabelecida a seguinte questão norteadora: “Qual o perfil das intervenções educativas voltadas para a prevenção da violência obstétrica?"

A coleta ocorreu no mês de março de 2021, no qual buscou-se publicações nas fontes de dados: Cumulative Index to Nursing and Allied Health Literature (CINAHL), Scopus Content Overview (SCOPUS), Literatura Latino-Americana e do Caribe em Ciências da Saúde (LILACS) e PubMed.

Realizou-se uma busca nos Descritores em Ciências da Saúde (DeCS)/Medical Subject Headings (MeSH) e elencados os descritores controlados, a saber: "educação", "educação em saúde", "obstetrícia", “education", "health education" e "obstetric". Também foram utilizadas as palavras-chave "violência obstétrica" e "obstetric violence". Para o cruzamento destes foram utilizados os operadores booleanos AND e OR.

Os critérios de inclusão foram: artigos que respondessem ao objetivo do estudo; que estivessem disponíveis na íntegra; sem delimitação de idioma; e publicados a partir de 2007, ano em que pela primeira vez a violência obstétrica foi oficialmente conceituada, mediante a 


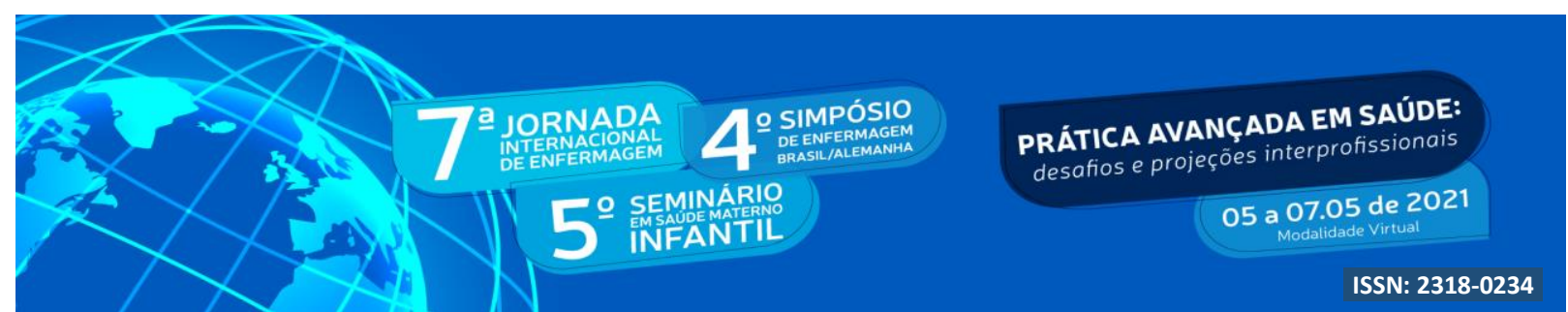

Lei Orgânica Sobre o Direito das Mulheres a uma Vida Livre de Violência (REPÚBLICA BOLIVARIANA DE VENEZUELA, 2007). Os critérios de exclusão foram: publicações em formato de editorial, carta ao editor, dissertação, tese, revisão da literatura e documentos duplicados.

Para minimizar o risco de viés, toda busca foi executada simultaneamente por dois pesquisadores, de forma independente, utilizando como suporte o software State of the Art through Systematic Review (StArt). Em sua interface foram inseridos o protocolo de pesquisa e os resultados das buscas nas fontes de dados, de modo a serem armazenados e organizados. No caso de divergências, um terceiro pesquisador foi consultado.

A princípio, foram encontradas 817 publicações. Após aplicação dos critérios de elegibilidade e leitura dos títulos e resumos, foram selecionados 47 artigos para leitura na íntegra. Após leitura criteriosa dos textos, 12 artigos foram escolhidos para compor a amostra final. Para um melhor entendimento do método aplicado foi construído um fluxograma conforme a figura 1.

Figura 1: Fluxograma do método aplicado. Natal, 2021

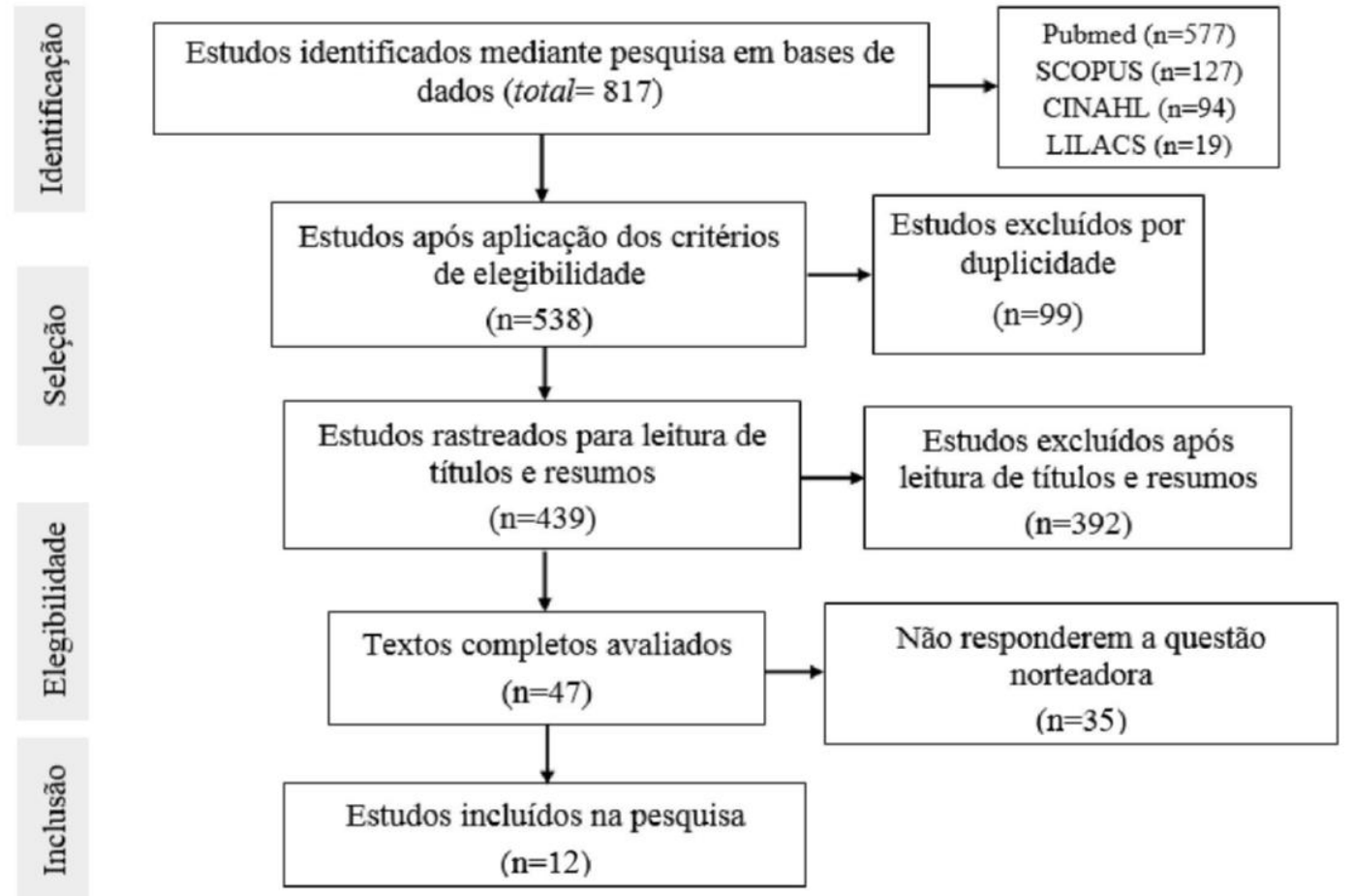

Fonte: Autoria própria 


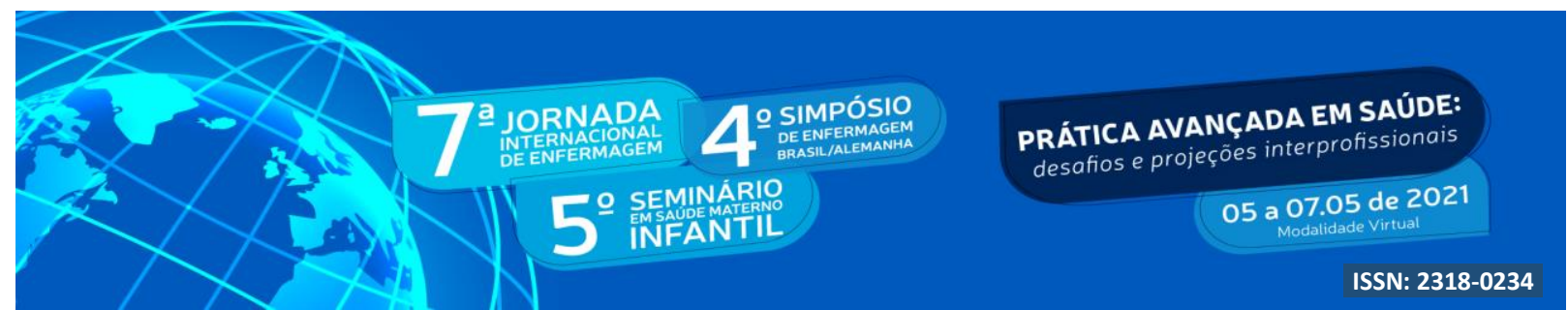

Os achados foram apresentados em uma tabela para descrever os artigos em termos de autoria, ano, público alvo, intervenção educativa, principais resultados, tipo de estudo e nível de evidência conforme o Joanna Briggs Institute (2013), que sugere uma classificação do tipo piramidal, em que no topo da pirâmide estão os estudos mais robustos do tipo 1, e na base da pirâmide se encontram os estudos com menor nível de evidência, do tipo 5.

\section{RESULTADOS E DISCUSSÃO}

Foram selecionados 12 estudos para compor a amostra da presente revisão. Destes, a maior parte foi publicada nos anos de 2020 (25\%), 2019 (25\%) e 2017 (25\%). Os estudos foram realizados em 8 locais diferentes, sendo no Brasil (33,3\%), Espanha (16,7\%), Qatar (8,3\%), Colômbia (8,3\%), México (8,3\%), Tanzânia (8,3\%), Sri Lanka $(8,3 \%)$ e Gauteng $(8,3 \%)$.

Em relação à abordagem metodológica, $66,7 \%$ foram do tipo experimental e 33,3\% do tipo observacional. Todos os estudos estavam disponíveis no idioma inglês, sendo que $25 \%$ apresentaram versão em português e 8,3\% na versão em espanhol.

A tabela 1 apresenta uma síntese dos dados dos estudos incluídos na revisão, posteriormente discutidos de acordo com a literatura pertinente.

Tabela 1. Síntese dos estudos incluídos na revisão. Natal, 2021

\begin{tabular}{|c|c|c|c|c|}
\hline Autor/Ano & $\begin{array}{l}\text { Intervenção } \\
\text { educativa }\end{array}$ & Público & Principais resultados & $\begin{array}{l}\text { Tipo de estudo/ } \\
\text { Nível de } \\
\text { evidência }\end{array}$ \\
\hline
\end{tabular}

\begin{tabular}{|c|c|c|c|c|}
\hline $\begin{array}{l}\text { Martínez- } \\
\text { Galiano et } \\
\text { al., } 2020\end{array}$ & $\begin{array}{l}\text { Programa de educação } \\
\text { em saúde pré-natal. }\end{array}$ & Puérperas. & $\begin{array}{l}\text { Mulheres passaram a } \\
\text { perceber atos de VO. }\end{array}$ & $\begin{array}{l}\text { Estudo } \\
\text { transversal/3 }\end{array}$ \\
\hline $\begin{array}{l}\text { Mena-Tudela } \\
\text { et al., } 2020\end{array}$ & $\begin{array}{l}\text { Seminário para alunos } \\
\text { da área da saúde. }\end{array}$ & Graduandos. & $\begin{array}{l}\text { Proporcionou reflexão e } \\
\text { identificação da VO. }\end{array}$ & $\begin{array}{l}\text { Estudo quase- } \\
\text { experimental/2 }\end{array}$ \\
\hline $\begin{array}{l}\text { Manrique; } \\
\text { Cuervo; } \\
\text { Castellanos, } \\
2020\end{array}$ & $\begin{array}{l}\text { Livreto educativo- } \\
\text { comunicativo sobre } \\
\text { parto humanizado. }\end{array}$ & $\begin{array}{l}\text { Gestantes e } \\
\text { familiares. }\end{array}$ & $\begin{array}{l}\text { Garante a compreensão } \\
\text { sobre os direitos das } \\
\text { mulheres e o parto } \\
\text { humanizado. }\end{array}$ & $\begin{array}{l}\text { Estudo de } \\
\text { validação/5 }\end{array}$ \\
\hline $\begin{array}{l}\text { Gray et al., } \\
2019\end{array}$ & $\begin{array}{l}\text { Vídeo abordando } \\
\text { ações de VO. }\end{array}$ & $\begin{array}{l}\text { Profissionais } \\
\text { da saúde. }\end{array}$ & $\begin{array}{l}\text { Mudança de percepções } \\
\text { da prática assistencial. }\end{array}$ & $\begin{array}{l}\text { Pesquisa } \\
\text { experimental/2 }\end{array}$ \\
\hline
\end{tabular}




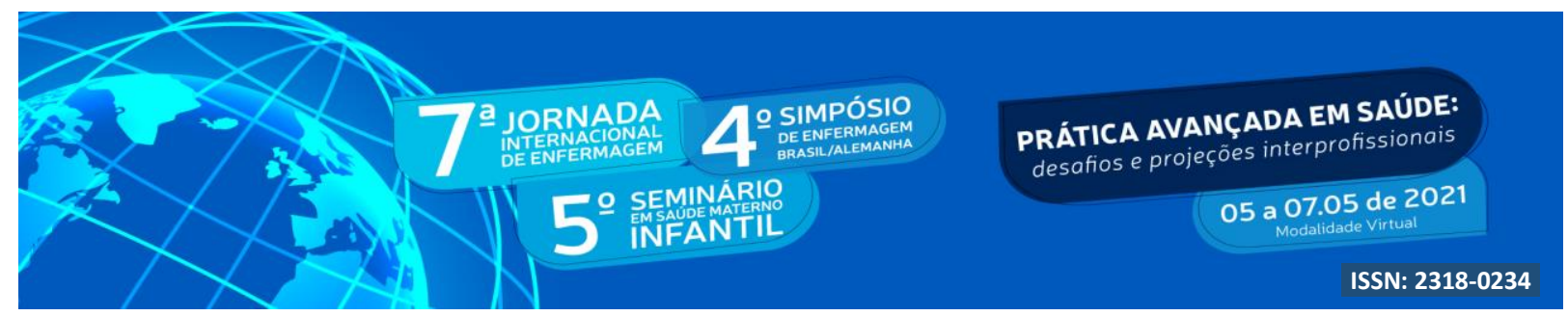

\begin{tabular}{lllll}
$\begin{array}{l}\text { Niy } \text { et al., } \\
2019\end{array}$ & $\begin{array}{l}\text { Foi realizado reuniões } \\
\text { entre grupos. }\end{array}$ & $\begin{array}{l}\text { Profissionais, } \\
\text { gestores e } \\
\text { puérperas. }\end{array}$ & $\begin{array}{l}\text { Falta de orientaçães } \\
\text { interferem na autonomia } \\
\text { da mulher. }\end{array}$ & $\begin{array}{l}\text { Projeto de } \\
\text { intervenção/4 }\end{array}$ \\
$\begin{array}{l}\text { Lansk } \text { et al., } \\
2019\end{array}$ & $\begin{array}{l}\text { Mobilização social } \\
\text { sentidos do nascer. }\end{array}$ & $\begin{array}{l}\text { Gestantes, } \\
\text { puérperas e } \\
\text { população. }\end{array}$ & $\begin{array}{l}\text { Aumentou a percepção } \\
\text { sobre ações de VO }\end{array}$ & $\begin{array}{l}\text { Estudo } \\
\text { transversal/3 }\end{array}$ \\
$\begin{array}{l}\text { Perera } \text { et al., } \\
2018\end{array}$ & $\begin{array}{l}\text { Treinamento de } \\
\text { atualização regular. }\end{array}$ & $\begin{array}{l}\text { Profissionais } \\
\text { da saúde. }\end{array}$ & $\begin{array}{l}\text { Cultivo da empatia e } \\
\text { reforço da ética. }\end{array}$ & $\begin{array}{l}\text { Pesquisa de } \\
\text { campo/3 }\end{array}$ \\
$\begin{array}{l}\text { Mselle; } \\
\text { Kohi; Dol, } \\
2018\end{array}$ & $\begin{array}{l}\text { Educação contínua dos } \\
\text { profissionais dos } \\
\text { serviços de saúde. }\end{array}$ & $\begin{array}{l}\text { Profissionais } \\
\text { da saúde. }\end{array}$ & $\begin{array}{l}\text { Conscientização sobre a } \\
\text { autonomia da mulher. }\end{array}$ & $\begin{array}{l}\text { Estudo } \\
\text { qualitativo/4 }\end{array}$ \\
$\begin{array}{l}\text { Verano } \text { et al., } \\
2017\end{array}$ & $\begin{array}{l}\text { Programas do } \\
\text { Ministério da Saúde. }\end{array}$ & $\begin{array}{l}\text { Profissionais } \\
\text { da saúde. }\end{array}$ & $\begin{array}{l}\text { Mudança de paradigmas } \\
\text { pelos profissionais jovens. }\end{array}$ & $\begin{array}{l}\text { Estudo } \\
\text { transversal/3 }\end{array}$ \\
$\begin{array}{l}\text { Santos; } \\
\text { Souza, } 2017\end{array}$ & $\begin{array}{l}\text { Cartilha sobre os } \\
\text { direitos das mulheres. }\end{array}$ & $\begin{array}{l}\text { Profissionais } \\
\text { de saúde. }\end{array}$ & $\begin{array}{l}\text { Tecnologia interativa no } \\
\text { processo de cuidado. }\end{array}$ & $\begin{array}{l}\text { Estudo } \\
\text { qualitativo/4 }\end{array}$ \\
$\begin{array}{l}\text { Fritz } \text { et al., } \\
2017\end{array}$ & $\begin{array}{l}\text { Simulação para } \\
\text { equipes } \\
\text { interprofissionais. }\end{array}$ & $\begin{array}{l}\text { Equipe de } \\
\text { saúde. }\end{array}$ & $\begin{array}{l}\text { Algumas mudanças foram } \\
\text { esporádicas. }\end{array}$ & $\begin{array}{l}\text { Ensaio clínico } \\
\text { randomizado/1 }\end{array}$ \\
$\begin{array}{l}\text { Apostilas, cartazes, } \\
\text { Brown } \text { et al., } \\
2007\end{array}$ & $\begin{array}{l}\text { Equipe de } \\
\text { saúde. }\end{array}$ & Redução de episiotomias. & $\begin{array}{l}\text { Ensaio piloto } \\
\text { randomizado /1 }\end{array}$ \\
\hline
\end{tabular}

Fonte: Autoria própria

As Nações Unidas incluem como Objetivos de Desenvolvimento Sustentável, a melhoria da saúde materna e a redução das taxas de morbimortalidade materno-infantil. (NAÇÕES UNIDAS, 2015). No entanto, para alcançar tais objetivos, torna-se imprescindível a utilização de ferramentas que previnam e reduzam os casos de VO, viabilizando o cuidado humanizado e de qualidade antes, durante e após o parto (DIAZ-TELLO, 2016).

A partir da análise dos estudos que compõem a revisão, observou-se a importante repercussão das práticas de educação em saúde para gestantes e puérperas. Diante da normalização da VO e da falta de orientações, as próprias mulheres se tornam incapazes de distinguir entre os atos de violação de seus direitos, das práticas assistenciais recomendadas, resultando na subnotificação dos casos abusivos e desrespeitosos (ABUYA et al., 2015).

Em consonância com este estudo, Mihret (2019) identificou que mulheres com acesso limitado à informações possuem $51 \%$ menos probabilidade em relatar atos de VO, quando comparado aquelas que frequentam o ensino médio e superior. Em pesquisa semelhante, 


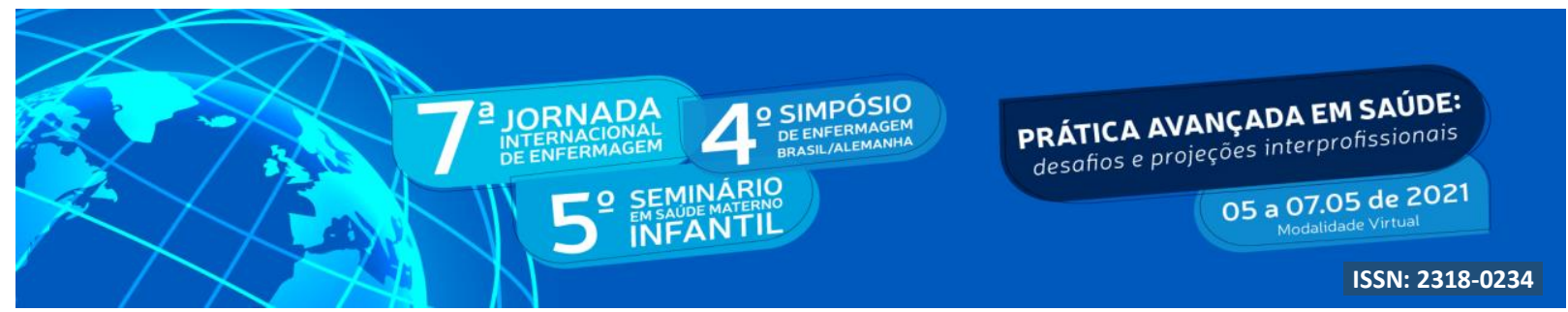

observou-se a associação entre o maior nível de instrução acerca dos direitos sobre o parto e nascimento e a maior prevalência de relatos de VO (GEBREMICHAEL et al.,2018).

Diante desse cenário, com o fornecimento de informações adequadas, as mulheres passam a identificar atos abusivos e desrespeitosos com maior facilidade, possibilitando a autonomia feminina e apropriação de seus direitos, de modo a favorecer a decisão de escolhas (SILVA et al., 2019). Ainda, a prévia orientação sobre os procedimentos realizados, aumenta a probabilidade de cumprimento de ações seguras e recomendadas, promovendo à mulher sentimentos positivos sobre seu parto (GEBREMICHAEL et al., 2018).

Sob outra perspectiva, ressalta-se que as intervenções educativas acerca dos direitos sobre os cuidados de maternidade e VO, repercutem positivamente para reflexão e conscientização da prática assistencial, quando direcionadas para graduandos e profissionais de saúde recém-formados. Como resultado, estes tornam-se capazes de identificar e prevenir ações abusivas e de desrespeito que, atualmente, são naturalizadas, corroborando com os achados da revisão (SADLER et al., 2016).

Dessa forma, as ações educativas configuram-se como principal instrumento para transformação do modo de pensar e produzir saúde, pela sua capacidade de gerar pensamento crítico-reflexivo e consequente mobilização dos indivíduos (PADILHA et al., 2018). Nessa direção, evidencia-se a gradual mudança de paradigmas acerca da assistência ao parto e nascimento, caracterizando a educação como uma ferramenta eficaz para a erradicação da VO (DIAZ-TELLO, 2016).

Em contrapartida, as intervenções analisadas geraram menores repercussões nos profissionais de saúde mais experientes, o que pode ser justificado pela natureza paradigmática e estrutural da VO (STONE, 2016). Nessa direção, a cultura do preconceito de gênero que restringe a mulher a um ser submisso, tem contribuído com a banalização e legitimação da VO, favorecendo o enraizamento dessa conduta ao longo da experiência profissional e dentro das instituições, contribuindo com maior resistência à mudanças (JARDIM; MODENA, 2018; BEGLEY et al., 2019).

Assim, vê-se que os efeitos das ações educativas podem não se sustentar ao longo do tempo, devido à falta de divulgação sobre a temática e apoio político e institucional. Logo, é preciso reconhecer que a prevenção da VO não se restringe ao âmbito da educação profissional, requer também articulação com o poder institucional e governamental, de modo 


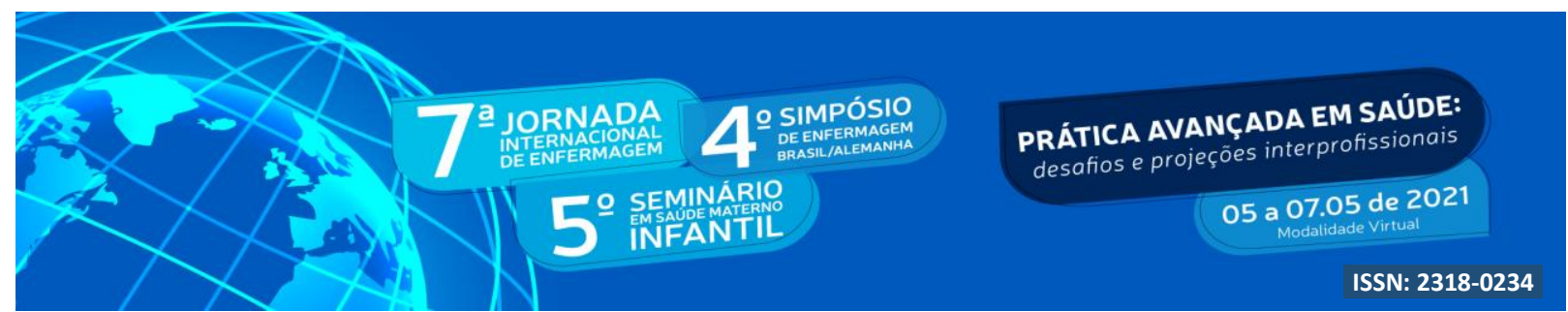

a garantir os direitos das usuárias, seja pela criação de normas e políticas ou pela responsabilização do cuidado humanizado (DIAZ-TELLO, 2016).

Corroborando com o presente estudo, uma pesquisa realizada com puérperas, identificou redução de 40-50\% na prevalência de atos de abuso e desrespeito, durante o trabalho de parto, após intervenção educativa institucional em conjunto com a população geral (ABUYA et al., 2015). Ainda, pesquisa afirma que a ampliação da educação em saúde traz impactos importantes para a divulgação da temática, oportunizando a mobilização social para promoção do cuidado humanizado (MSELLE; KOHI; DOL, 2018; SILVA et al., 2019).

Por fim, ressalta-se a limitação do presente estudo pela escassez de evidências disponíveis acerca do monitoramento das intervenções educativas, restringindo a análise sobre seus impactos ao longo do tempo. No entanto, esta pesquisa mostra-se relevante pela necessidade de conscientização social acerca dos riscos da assistência abusiva e desrespeitosa às mulheres, por meio do fornecimento de dados e comprovantes de sua ocorrência.

\section{CONCLUSÃO}

O estudo mostrou que as intervenções educativas impactam diretamente na construção do pensamento crítico e reflexivo acerca da real problemática da violência obstétrica. Quando as ações são direcionadas às mulheres, vê-se que estas se empoderam de seus direitos na medida em que compreendem os significados atribuídos à violência obstétrica, o que contribui para promoção da autonomia. Ainda, observa-se a gradual mobilização dos provedores de cuidados para coibição de práticas abusivas e desrespeitosas.

Vale destacar a contribuição do presente estudo para a assistência materno-infantil, por proporcionar o entendimento das lacunas no conhecimento, fornecendo subsídios que viabilizem a construção de intervenções educativas eficazes e direcionadas, com embasamento científico, capazes de prevenir casos de violência obstétrica e promover assistência holística durante o ciclo gravídico-puerperal.

\section{REFERÊNCIAS}

ABUYA, T. et al. Exploring the prevalence of disrespect and abuse during childbirth in Kenya. PLoS One, v. 10, n. 4, p. e0123606, 2015. 


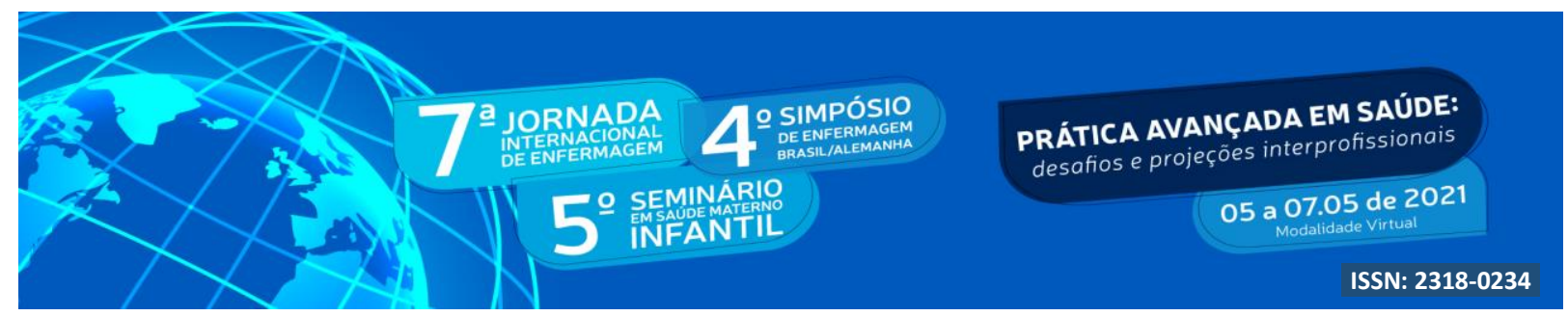

BEGLEY, K. et al. Shared decision-making in maternity care: Acknowledging and overcoming epistemic defeaters. J Eval Clin Pract., v. 25, n. 6, p. 1113-1120, 2019.

BROWN, H. et al. Promoting childbirth companions in South Africa: a randomised pilot study. BMC Medicine, v. 5, n. 7, p. 1-8, 2007.

DIAZ-TELLO, F. Invisible wounds: obstetric violence in the United States. Reproductive Health Matters, v. 24, n. 47, p. 56-64, 2016.

FRITZ, J. et al. Can a simulation-based training program impact the use of evidence based routine practices at birth? Results of a hospital-based cluster randomized trial in Mexico. PLoS ONE, v. 12, n. 3, p. e0172623, 2017.

GRAY, T. et al. Obstetric violence: Clinical staff perceptions from a video of simulated practice. European Journal of Obstetrics \& Gynecology and Reproductive Biology, v. 1, p. 1-5, 2019.

GEBREMICHAEL, M.W. et al. Mothers' experience of disrespect and abuse during maternity care in northern Ethiopia. Glob Health Action., v. 11, suppl 3, p. 1465215, 2018.

JARDIM, D. M. B; MODENA, C. M. Obstetric violence in the daily routine of care and its characteristics. Rev Lat Am Enfermagem, v. 26, p. e3069, 2018.

JOANNA BRIGGS INSTITUTE. JBI Levels of Evidence. 2013. Disponível em: <https://joannabriggs.org/\#>. Acesso em: 10 set 2020.

LANSKY, S. et al. Obstetric violence: influences of the Senses of Birth exhibition in pregnant women childbirth experience. Ciência \& Saúde Coletiva, v. 24, n. 8, p. 2811-2823, 2019.

MANRIQUE, F. M. C; CUERVO, G. M. N; CASTELLANOS, A. D. L. Elaboration and Validation of a Booklet on Humanized Childbirth. Enfermería Global., v. 19, n. 4, 64-84, 2020 .

MARTÍNEZ-GALIANO, J. M. et al. The magnitude of the problem of obstetric violence and its associated factors: A cross-sectional study. Women and Birth, p. 1-9, 2020.

MENA-TUDELA, D. et al. Changes in health sciences students' perception of obstetric violence after an educational intervention. Nurse Education Today, v. 88, p. 1-7, 2020.

MENEZES, F. R. et al. The obstetric nursing residents' view on obstetric violence in institutions. Interface (Botucatu), v. 24, p. e180664, 2020.

MIHRET, M.S. Obstetric violence and its associated factors among postnatal women in a Specialized Comprehensive Hospital, Amhara Region, Northwest Ethiopia. BMC Res Notes., v. 12 , p. $600,2019$. 


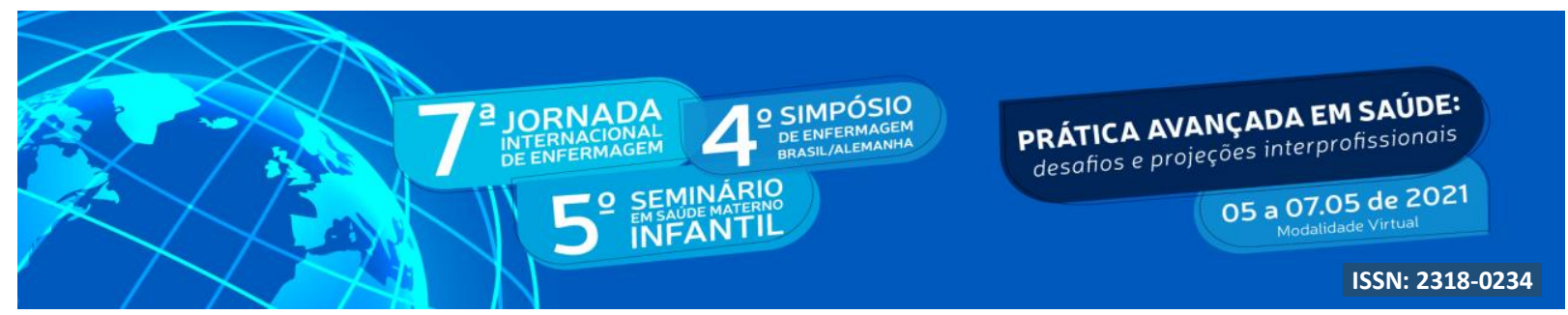

MSELLE, L. T; KOHI, T. W; DOL, J. Barriers and facilitators to humanizing birth care in Tanzania: findings from semistructured interviews with midwives and obstetricians.

Reproductive Health, v. 15, n. 137, 2018.

NAÇÕES UNIDAS BRASIL. Objetivo de Desenvolvimento Sustentável 3. 2015.

Disponível em: <https://brasil.un.org/pt-br/sdgs/3>. Acesso em: 8 mar 2021.

NIY, D. Y. et al. Overcoming the culture of physical immobilization of birthing women in Brazilian healthcare system? Findings of an intervention study in São Paulo, Brazil. Interface (Botucatu), v. 23, p. e180074, 2019.

PADILHA, R. Q. et al. Principles of clinical management: connecting management, healthcare and education in health. Ciência \& Saúde Coletiva, v. 23, n. 12, p.4249-4257, 2018.

PERERA, D. et al. 'When helpers hurt': women's and midwives' stories of obstetric violence in state health institutions, Colombo district, Sri Lanka. BMC Pregnancy and Childbirth, v. 18, n. 211, p. 1-12, 2018.

REPÚBLICA BOLIVARIANA DE VENEZUELA. Ley Orgánica sobre el derecho de las mujeres a una vida libre de violencia. GORBV, 2007.

SADLER, M. et al. Moving beyond disrespect and abuse: addressing the structural dimensions of obstetric violence. Reproductive Health Matters, v. 24, p. 47-55, 2016.

SANDO, D. et al. Methods used in prevalence studies of disrespect and abuse during facility based childbirth: lessons learned. Reprod Health., v. 14, n. 127, p. 1-18, 2017.

SANTOS, A. L. M; SOUZA, M. H. T. Elaboration of new technologies in nursing: use of a prevention booklet. Rev enferm UFPE on line., v. 11, n. 10, p. 3893-3898, 2017.

SILVA, W. B. et al. Educação em saúde acerca da prevenção da violência obstétrica: relato de experiência. Electronic Journal Collection Health, v.11, n. 14, p. e1163, 2019.

STONE, P. K. Biocultural perspectives on maternal mortality and obstetrical death from the past to the present. American Journal of Physical Anthropology, v. 159, p. 150-171, 2016.

ORGANIZAÇÃO MUNDIAL DE SAÚDE. Prevenção e eliminação de abusos, desrespeitos e maus tratos durante o parto em instituições de saúde. Genebra: OMS, 2014.

VERANO, J. B. et al. Shifting the paradigm to an obstetric humanized care: The disparities at a tertiary care hospital in the midwest of Brazil. Clinical and Experimental Obstetrics \& Gynecology, v. 46, n. 4, p. 521-525, 2019. 